Schmerz 2017· 31:431-432

https://doi.org/10.1007/s00482-017-0243-9

Online publiziert: 24. August 2017

(c) Deutsche Schmerzgesellschaft e.V. Published by Springer Medizin Verlag GmbH - all rights reserved 2017

CrossMark

\author{
W. Häuser ${ }^{1,2} \cdot$ M. Keidel $\left.\right|^{3}$ \\ ${ }^{1}$ Innere Medizin 1, Klinikum Saarbrücken, Saarbrücken, Deutschland \\ ${ }^{2}$ Klinik für Psychosomatische Medizin und Psychotherapie, Technische Universität München, München, \\ Deutschland \\ ${ }^{3}$ Neurologische Klinik, Campus Bad Neustadt, Rhön-Klinikum, Bad Neustadt, Deutschland
}

\title{
Gemeinsam klug entscheiden in der Schmerzmedizin
}

In diesem Heft stellen drei Übersichtsarbeiten psychotherapeutische und psychologische Verfahren in der Schmerzmedizin vor: Entspannungsverfahren und Verhaltenstherapie bei der Migräne [3] und kognitiv-perzeptive Ansätze [4] bzw. „eye movement desensitization and reprocessing" (EMDR; [5]) bei chronischen Schmerzen. Beim Lesen dieser Beiträge können sich für den Praktiker/Kliniker folgende Fragen ergeben: Wie wirksam und sicher sind die Verfahren? Sind diese Verfahren bei meinen Patienten indiziert? Sind diese Verfahren für mich und meine Patienten verfügbar? Falls ja, wie kann ich meine Patienten über diese Verfahren und ihre Alternativen informieren? Wie werden diese Verfahren von meinen Patienten angenommen?

Diese möglichen Fragen eines lesenden Praktikers berühren zentrale Themen des Deutschen Schmerzkongresses 2017: Gemeinsam entscheiden = partizipative Entscheidungsfindung und Klug entscheiden $=$ „Choosing wisely“. Das Tagungsthema des Deutschen Schmerzkongresses 2017 „Gemeinsam entscheiden" bezieht sich auch auf die Initiative „Klug entscheiden“ der Arbeitsgemeinschaft der Wissenschaftlichen Medizinischen Fachgesellschaften (AWMF) in Deutschland. „Gemeinsam Klug Entscheiden“ ist eine Qualitätsoffensive der Wissenschaftlichen Medizinischen Fachgesellschaften unter dem Dach der AWMF und zielt auf die Verbesserung der Versorgungsqualität durch ausgewählte Empfehlungen $\mathrm{zu}$ prioritären Themen. Sie betont die Gemeinsamkeit der Fachgesellschaften in der AWMF, die gemeinsame fach- und berufsgruppenübergreifende Versorgung und die gemeinsame Entscheidungsfindung von Arzt und Patient. Sie stellt Patienten/ Versorgungsaspekte zu Erkrankungen in den Mittelpunkt und nicht Fachgebiete. Sie soll Patienten zur Teilhabe im Rahmen der partizipativen Entscheidungsfindung befähigen und eine wissenschaftlich und ethisch begründete Entscheidungsfindung als Antwort auf eine zunehmend marktwirtschaftliche Orientierung des Gesundheitssystems ermöglichen [1].

Die Deutsche Gesellschaft für Innere Medizin (DGIM) hat eine Vorreiterrolle bei der Initiative „Klug entscheiden“ übernommen. Ihre Initiative „Klug entscheiden“ identifiziert wichtige evidenzbasierte Maßnahmen der Diagnostik und Therapie, die häufig nicht fachgerecht erbracht werden. Nach DGIM-Kriterien ist Überversorgung (überflüssige Leistung) definiert als eine diagnostische/ therapeutische Maßnahme, die häufig durchgeführt wird, obwohl sie nachweislich nicht nutzbringend oder sogar schädlich ist. Hieraus resultiert eine Negativempfehlung. Unterversorgung (unterlassene Leistung) ist eine diagnostische/therapeutische Maßnahme, die häufig nicht durchgeführt wird, obwohl sie nachweislich für den Patienten sinnvoll ist. Hieraus resultiert eine Positivempfehlung. Über- und Unterversorgung sind einerseits charakterisiert durch die vorhandene Evidenz, andererseits durch die Häufigkeit der Fehlversorgung. Die Empfehlungen wurden evidenz- und konsensbasiert unter Einbeziehung von Patientenvertretern erstellt [2]. Inzwi- 
schen haben alle 12 Fachgesellschaften der internistischen Schwerpunkte beziehungsweise assoziierten Fachgesellschaften der DGIM je fünf Positiv- und Negativempfehlungen für ihr Teilgebiet erstellt. Alle Empfehlungen wurden im Deutschen Ärzteblatt veröffentlicht. Einige Positiv- und Negativempfehlungen, z. B. der Rheumatologie und Palliativmedizin, sind auch für Schmerzmediziner von großer Relevanz.

Von besonderer Bedeutung in der Schmerzmedizin sind eine ausführliche Kommunikation mit den Patienten unter Einbeziehung meinungsbeeinflussender Bezugspersonen sowie eine verständliche Information über mögliche Therapieoptionen, die eine gemeinsam getragene „kluge“ Entscheidungsfindung bezüglich einer aussichtsreichen Behandlung ermöglichen. Die patientenseitige zuversichtliche Erwartung eines Therapieerfolgs und die Motivation zur selbstwirksamen und aktiven Teilnahme an der Therapie hängen wesentlich von einem vertrauensvollen Arzt-PatientenKonsens in der Entscheidungsfindung der (diagnostischen) und therapeutischen Vorgehensweisen $\mathrm{ab}$.

Wenn der Leser dieses Editorials mehr zu „Gemeinsam Klug Entscheiden in der Schmerzmedizin“ erfahren will, ist er auf dem Deutschen Schmerzkongress vom 11.10.-14.10.2017 in Mannheim herzlich willkommen. In ihrem Festvortrag wird Frau Professor Dr. med. Ina Kopp, Leiterin des AWMF-Instituts für Medizinisches Wissensmanagement und Vorsitzende des Guidelines International Network, die Chancen und Gefahren von evidenzbasierter Medizin und von Leitlinien sowie die Relevanz der Gemeinsam-Klug-Entscheiden-Initiativen verschiedener AWMF-Fachgesellschaften für die Schmerzmedizin darstellen. Ihr Festvortrag wie auch ein Symposium (u. a. mit einer Vertreterin von SchmerzLOS) werden kritisch diskutieren, wie es um die partizipative Entscheidungsfindung in der deutschen Schmerzmedizin bestellt ist und wie diese im klinischen Alltag verbessert werden kann.
Wir freuen uns über Ihre Teilnahme am Deutschen Schmerzkongress 2017 in Mannheim.

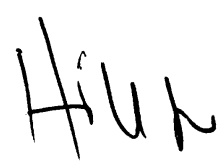

Winfried Häuser

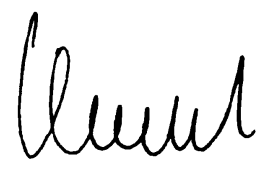

Matthias Keidel

\section{Korrespondenzadresse}

\section{W. Häuser}

Innere Medizin 1, Klinikum Saarbrücken

Saarbrücken, Deutschland

whaeuser@klinikum-saarbruecken.de

Interessenkonflikt. W. Häuser und M. Keidel geben an, dass kein Interessenkonflikt besteht.

\section{Literatur}

1. Arbeitsgemeinschaft der Medizinisch Wissenschaftlichen Fachgesellschaften AWMF (2015) Gemeinsam klug entscheiden. www. awmf.org/medizin-versorgung/gemeinsamklug-entscheiden.html. Zugegriffen: 27. Juli 2017

2. HasenfußG, Märler-Herrman $E$, Hallek M, Fölsch R (2016) Initiative „Klug entscheiden“. Gegen Unterund Überversorgung. Dtsch Ärztebl 11:A 600-A 603

3. Kropp P (2017) Entspannungsverfahren und verhaltenstherapeutische Interventionen zur Behandlung der Migräne - Leitlinie der Deutschen Migräne- und Kopfschmerzgesellschaft. Schmerz. https://doi.org/10.1007/s00482-017-0214-1

4. Storz C (2017) Kognitiv-perzeptive Ansätze bei chronischen Schmerzen.Schmerz.https://doi.org/ 10.1007/s00482-017-0229-7

5. Wicking M(2017) EMDRals psychotherapeutischer Ansatz in der Schmerztherapie. Schmerz. https:// doi.org/10.1007/s00482-017-0231-0
Auch online Zugang zu allen Beiträgen Ihres Zeitschriftenabonnements

Wussten Sie, dass Sie als Abonnent dieser Zeitschrift automatisch online Zugriffsrechte auf das gesamte Beitragsarchiv haben?

Der Zugang zu Ihrer Online-Bibliothek ist im Abonnement Ihrer Zeitschrift inbegriffen. Für den Zugang müssen Sie sich lediglich einmal über www.springermedizin.de/register registrieren.

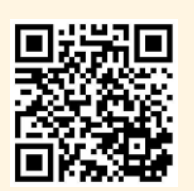

Über diesen QR-Code schnell und einfach registrieren

Bitte nutzen Sie für die Registrierung Vorund Nachname und Lieferadresse wie beim Abonnement der Zeitschrift (siehe Adressaufkleber auf Ihrem Heft). So kann im System die Zugehörigkeit zu Ihrer Zeitschrift sichergestellt werden.

Aufgrund des Heilmittelwerbegesetzes dürfen die Inhalte der Website nur medizinischen Fachkreisen zur Verfügung gestellt werden. Bei der Anmeldung bitten wir Sie deshalb einen Berufsnachweis vorzulegen.

Bei Medizinern mit Mitgliedschaft in der deutschen Ärztekammer reicht die einheitliche Fortbildungsnummer (EFN). Alternativ schicken Sie eine Bestätigung des Arbeitgebers, Studiennachweis oder andere Zeugnisse ganz unkompliziert an kundenservice@springermedizin.de.

Mit Benutzername und Passwort haben Sie außerdem Zugang zu den freien Inhalten auf den Seiten von: https://www.springermedizin.de/ https://www.aerztezeitung.de/

Sollten Fragen oder Probleme auftauchen, wenden Sie sich einfach an Ihren Kundenservice:

kundenservice@springermedizin.de 